\title{
GRADO DE EQUIVALENCIA ENTRE LA PROTECCIÓN DE LOS DATOS PERSONALES Y EL DERECHO DE ACCESO A LA INFORMACIÓN PÚBLICA
}

["Level equivalence between the protection of personal information and access to public information: elements to overcome the tension between these warranties"]

\section{Francisco Javier Sanz Salguero*}

\begin{abstract}
RESUMEN
A partir de la entrada en vigencia de la Ley de transparencia, ha sido permanente la tensión en la labor de conciliación entre el acceso a la información pública y la protección de los datos personales. En este documento, analizamos un tema esencial que debe ser tenido en cuenta a fin de superar la tensión entre estas garantías: su
\end{abstract}

\begin{abstract}
From the entry into force of the Transparency and Access to Public Information Act, there has been a permanent tension in the work of reconciliation among the access to public information and the protection of personal data. In this paper, we discuss a key issue that must be taken into account in order to overcome
\end{abstract}

RECIBIDO el 2 de noviembre 2016 y APROBADO el 24 de marzo de 2017

* Doctor en Derecho por la Pontificia Universidad Católica de Valparaíso. Abogado Universidad Externado de Colombia. Dirección postal: Avenida Brasil 2950, Valparaíso, Chile. Dirección electrónica: fjsanzsalguero@hotmail.com. El autor agradece al Prof. Dr. Alan Bronfman Vargas, por los aportes realizados durante el desarrollo de este artículo.

El presente artículo, se ha redactado dentro del marco de la tesis doctoral titulada "La solicitud de acceso a la información y tutela de los datos personales del tercero, dentro del marco de aplicación de la Ley de transparencia de la función pública y de acceso a la información de la Administración del Estado", investigación desarrollada por el autor. 
carácter de derechos fundamentales con relevancia equivalente.

Palabras Claves

Transparencia - Acceso a información pública - Datos personales the tension between these guarantees: the character of fundamental rights equivalent relevance.

KEYWORDS

Transparency - Access to public information - Personal data.

\section{INTRODUCCIÓN}

En el contexto jurídico chileno, la preocupación por el resguardo de la vida privada y, consecuentemente, de los datos de carácter personal, han sido temas de permanente regulación normativa. En este orden de ideas, ya el primer intento por reconocer (eso sí, de manera incipiente) una cierta protección a la esfera privada de las personas, se remonta al Reglamento Constitucional Provisorio de 1812, pasando por textos como las constituciones de 1818, 1828, 1833 y 1925, hasta llegar al Acta Constitucional No 3 del 11 de septiembre de 1976 (primera regulación en consagrar la protección de la vida privada de las personas en el ordenamiento constitucional chileno), antecedente directo e inmediato de la regulación que la Carta Política de 1980 hace respecto a la tutela de este derecho, culminado este recorrido histórico-normativo con la promulgación de la ley No 19.628 de 1999, estatuto que en su trámite parlamentario surgió bajo la pretensión de tutelar la vida privada, pero que luego de un largo trámite legislativo terminó enfocándose en la protección de los datos de carácter personal. Casi diez años después de la entrada en vigencia de la ley No 19.628, el legislador promulgó la "Ley de transparencia de la función pública $y$ de acceso a la información de la Administración del Estado"(aprobada por el artículo $1^{\circ}$ de la Ley No 20.285 del 2008), también conocida como "Ley de transparencia", estatuto en el que se da tratamiento al régimen de acceso a la información pública. En este sentido y como nos recuerda Enrique Rajevic, ambos cuerpos legales regulan la información, pero desde diversas aristas ${ }^{1}$ : el primero resguarda la información que concierne a personas naturales identificadas o identificables (es decir, los datos personales) buscando garantizar que sus titulares sean quienes decidan sobre su uso. Entre tanto, el ámbito del segundo es la información que obra en poder de

Rajevic Mosler, Enrique, Protección de datos y transparencia en la administración pública chilena: Inevitable y deseable ponderación /en/ VV.AA. Reflexiones Sobre el Uso y Abuso de los Datos Personales en Chile (Santiago, Expansiva, 2011), p. 139. 
los órganos del Estado (la que puede incluir datos de carácter personal) con la pretensión de favorecer su conocimiento por parte de los ciudadanos.

$\mathrm{Al}$ respecto y según subraya el citado autor, la protección de los datos personales ampara la intimidad y la autodeterminación informativa, mientras que la transparencia administrativa favorece la probidad y potencia la participación ciudadana ${ }^{2}$. Sin embargo, dentro del marco de aplicación de la normativa encargada del régimen de acceso a la información pública, surge la necesidad de establecer qué tratamiento debe otorgarse a la protección de los datos de carácter personal en su relación con el acceso a la información, identificando el término "acceso" como un mecanismo central en aras del logro de la transparencia en lo público. En efecto, nos encontramos ante dos bienes jurídicos (tutela de la información de naturaleza personal y derecho de acceso) reconocidos en el ámbito legal y, por ende, potencialmente antagónicos, tensión manifestada (entre otros factores) con el gran número de decisiones de amparo emitidas por el Consejo para la Transparencia desde su creación, decisiones originadas dentro del estudio de la relación entre los mencionados bienes jurídicos ${ }^{3}$.

La necesidad de establecer el tratamiento que debe otorgarse a la relación entre la protección de los datos personales y el derecho de acceso a la información de naturaleza pública (dentro del marco de aplicación de la "Ley de transparencia"), plantea una dificultad adicional cuando la esfera de la información personal a la que pretende accederse, afecta los datos de una persona diferente del solicitante. Paralelamente, si la información nominativa es requerida por el propio titular de los datos, ya entraríamos en la órbita del denominado habeas data.

Proponer una solución adecuada a fin de lograr el equilibrio en la relación entre el acceso a la información pública (como herramientas de

${ }^{2}$ Ibíd.

${ }^{3}$ El Consejo para la Transparencia ha debido ejercer la atribución consagrada en el artículo 33 letra m) de la "Ley de transparencia", aprobada por el artículo $1^{\text {o }}$ de la Ley No 20.285 de 2008, consistente en velar por el debido cumplimiento de la Ley No 19.628, sobre protección de datos de carácter personal por parte de los órganos de la Administración del Estado. A este respecto y dentro de su labor jurisprudencial, observamos por ejemplo que entre agosto de 2010 y marzo de 2011, el número de decisiones de fondo que aplicaron precisamente la citada Ley sobre protección de datos fue de 132, representando más del $27 \%$ de la totalidad de las providencias de fondo proferidas por este organismo. Ésta estadística confirma la importancia que ha tenido el estudio de la relación entre el derecho de acceso de la información pública (como instrumento central en aras del logro de la transparencia en lo público) y la tutela de los datos de carácter personal para el Consejo. Véase: Consejo para la Transparencia, Protección de datos personales (Santiago, Consejo para la Transparencia, 2011), p. 38. 
control social en un Estado de derecho) y el resguardo de los datos de carácter personal, es una labor que exige un estudio sistemático en diversas áreas de la doctrina, la legalidad y la jurisprudencia, tanto en el ámbito del derecho interno como del derecho comparado ${ }^{4}$. Precisamente, este artículo abarca una de las esferas que deben ser tenidas en cuenta dentro de la búsqueda del equilibrio en la relación entre el derecho de acceso y la protección de la información personal, entendiendo este equilibrio como el máximo alcance de los bienes jurídicos concernidos hasta su nivel de desarrollo compatible: desde una perspectiva que incluya tanto las normas de derecho interno, al igual que la doctrina y la jurisprudencia (especialmente la originada en organismos internacionales), en este trabajo examinamos si la protección de los datos personales y el derecho de acceso a la información pública son derechos fundamentales con relevancia equivalente. A fin de complementar el análisis anterior, concluimos el presente artículo proponiendo el mecanismo para resolver el conflicto entre personas que invocan, simultáneamente, sus derechos de naturaleza iusfundamental.

\section{EQUIVALENCIA NORMATIVA ENTRE LA PROTECCIÓN DE LOS DATOS PERSONALES Y EL DERECHO DE ACCESO A LA INFORMACIÓN PÚBLICA}

El estudio de la relación entre la protección de los datos personales y el derecho de acceso a la información pública, plantea un interrogante central: ¿estos son derechos fundamentales con relevancia equivalente? En este contexto, si se concluye que al comparar los bienes jurídicos materia de análisis (es decir, si se contrasta la protección de los datos personales con el derecho de acceso a la información pública) se observa un mayor o menor grado de jerarquía normativa de uno respecto al otro, entonces la labor de conciliación entre el acceso a la información pública y la protección de los datos de carácter personal (particularmente, los datos nominativos de terceros) será mucho menos compleja, ya que uno de los derechos (por virtud de la supremacía normativa) primará sobre el otro. Por el contrario, si al contrastar los derechos se concluye que poseen una relevancia equivalente, este trabajo de conciliación será más exigente.

En este orden ideas, resolver la pregunta formulada implica recorrer

${ }^{4}$ Sobre el particular y para tener en cuenta SAnz Salguero, Francisco Javier, Relación entre la protección de los datos personales y el derecho de acceso a la información pública dentro del marco del derecho comparado, en Revista Ius et Praxis 22 (2016) 1, pp. 323-376, trabajo en el cual, desde la perspectiva del derecho comparado, analizamos otros temas que deben ser tenidos en cuenta en la búsqueda del equilibro en la relación entre el derecho de acceso y la protección de la información personal. 
los siguientes pasos: en primer lugar, repasar los diferentes sentidos que se otorga al concepto de derecho fundamental (distinguiéndolo además de la noción de derecho fundamental constitucional); en segundo término, identificar la naturaleza jurídica de la protección de la información personal dentro del marco de la CPol. y, en tercer lugar, identificar la naturaleza jurídica del derecho de acceso a la información pública dentro de la esfera de la Carta de Derechos. A continuación entonces, llevaremos a cabo la labor propuesta.

\section{Puntos de vista en relación a la noción de derecho fundamental}

a) Actualmente, reconocemos una pluralidad de sentidos para la expresión derechos fund a mentales, formulándose tres alternativas para definir dicha expresión: $i$ ) en un sentido general, la citada expresión se usa para aludir aquellos derechos de la persona que han recibido consagración positiva, en particular a nivel constitucional; $i$ ) otra postura, identifica estos derechos como aquellos de los que es titular el hombre, no por graciosa concesión de las normas positivas, sino con anterioridad e independientemente de ellas y por el mero hecho de ser hombre, tratándose entonces de aquellos atributos vinculados directamente a la dignidad humana, atributos que constituyen un conjunto limitado en su extensión sólo a aquellos que son condición para la satisfacción de las necesidades inherentes a una vida digna; y iii) como tercera alternativa, se sugiere identificar estos de r e ch o s fund a m e n t a l es como aquellos derechos subjetivos que corresponden universalmente a todos los seres humanos, al poseer el status de personas con capacidad de obrar, de ciudadanos 5 .

b) Una solución frente a esta pluralidad de sentidos de la expresión de rech os fund a m en tales, sugiere distinguir entre un aspecto formal y un aspecto material del carácter fundamental de estos derechos. El aspecto formal del carácter fundamental se encontraría asociado a su constitucionalización lo que implicaría, en palabras de Gomes Canotilho ${ }^{6}$ i) que los preceptos que consagran derechos fundamentales, en cuanto preceptos fundamentales, gozan de una posición de supremacía en el ordenamiento jurídico; ii) que en cuanto a preceptos constitucionales, se ven afectados por los requisitos especiales de la reforma constitucional; iii) que como preceptos que dan origen a normas vinculantes, constituyen exigencias materiales del ejercicio de las competencias de los órganos públicos.

c) El aspecto material del carácter fundamental alude a la naturaleza

5 Aldunate Lizana, Eduardo, Derechos fundamentales, (Santiago, Legal Publishing, 2008), pp. 47 s.

6 Gomes Canotilho, José Joaquim, Direito Constitucional e Teoría da Constituição (5a edición, Almedina, Cohimbra, Portugal, 2002), p. 377. 
de ciertos derechos como elementos constitutivos del orden político, se encuentren o no consagrados en el texto constitucional. Conforme a lo anterior, cuando estos derechos fundamentales en sentido material se encuentran insertos en el texto constitucional, a partir de ellos se puede construir una teoría de límites implícitos al poder constituyente derivado; al contrario, cuando estos no se encuentran consagrados en una carta política, la idea de derechos fundamentales en sentido material justifica la denominada cláusula abierta de los derechos fundamentales, haciendo referencia a la apertura de fuentes para considerar, como parte del ordenamiento constitucional, tales derechos aun cuando no están incorporados en el texto de la Constitución. Es pertinente aclarar que la noción de cláusula abierta no implica una habilitación ilimitada al intérprete u órgano de la jurisdicción constitucional, para revelar un catálogo de derechos fundamentales implícitos en un texto normativo: solamente derechos cuya materialidad fundamental sea demostrable, pueden poseer tal calidad ${ }^{7}$.

d) Formulada la distinción entre un aspecto formal y un aspecto material del carácter fundamental de los derechos en estudio, nos corresponde distinguir las expresiones "derecho fundamental" y "derecho fundamental constitucional". En primer lugar, debemos afirmar que (a diferencia de la noción de derecho fundamental) el concepto de derechos constitucionales es mucho más acotado y menos ambiguo, ya que alude siempre a los derechos contenidos en un texto constitucional (generalmente un catálogo o listado de derechos), el que puede intentar extenderse a todos los derechos esenciales, o bien excluir algunos, admitiendo la posibilidad de incluir a otros que no pueden ser calificados ni como derechos esenciales ni como derechos fundamentales en un sentido material (por ejemplo, el derecho a indemnización por error judicial). Con base en lo anterior, podemos identificar la expresión derechos fundamentales constitucionales con el concepto de derechos fundamentales en su aspecto formal, es decir, derechos fundamentales reconocidos expresamente en el texto constitucional ${ }^{8}$.

e) Para finalizar y acorde con explicado, la doctrina opina mayoritariamente que el catálogo del artículo 19 de la Constitución (catálogo en el cual son consagrados los derechos de carácter fundamental) no es taxativo, admitiéndose así la posibilidad de que existan derechos fundamentales (en el sentido de esenciales, inherentes a la persona) no consagrados en la Carta Política?.

\footnotetext{
7 Aldunate Lizana cit. (n. 5), p. 49.

${ }^{8}$ Ibíd., p. 50.

${ }^{9}$ Ibíd., p. 345.
} 


\section{Naturaleza jurídica de la protección de los datos de carácter personal} dentro del marco de la Constitución Politica

En lo relativo a la protección de los datos de carácter personal, la tutela de este tipo de información es considerada como una derivación del derecho a la intimidad ${ }^{10}$. Para la doctrina, ésta protección es un derecho de tercera generación ${ }^{11}$, dotado de autonomía ${ }^{12}$. No se trataría entonces del "puro derecho a ser dejado solo, en la formulación decimonónica del derecho a la intimidad (the right to be let alone), sino del derecho a la autodeterminación informativa, esto es, el derecho de las personas a controlar sus datos personales, incluso si éstos no se refieren a su intimidad"13.

Para el caso chileno, durante el trabajo de consagración del derecho a la vida privada, dentro del proceso de redacción de la Carta Política de 1980 y la reforma constitucional del 2005, se discutió la adopción de expresiones como "intimidad" o "privacidad", estableciéndose finalmente el actual artículo 19 No $4^{\circ}$ CPol., norma en la que se reconoce expresamente el "respeto y protección a la vida privada y a la honra de la persona y su familia"14. Observamos entonces que en Chile, claramente, la vida privada posee el carácter de derecho fundamental constitucional (o derecho fundamental en su aspecto formal), al ser reconocido expresamente en el texto de la Constitución. En este orden de ideas, los datos personales representan un tipo de información que hace parte de la vida privada, por lo que la protección de la vida privada concierne y beneficia directamente a la información de naturaleza personal.

Ya a nivel normativo, el estatuto legal más importante aprobado hasta la fecha en materia de protección de la vida privada, la intimidad y los datos personales ${ }^{15}$, concretamente la Ley No 19.628 (que aparece titulada como:

${ }^{10}$ Rajevic Mosler, Enrique, cit. (n. 1), p 142.

${ }^{11}$ Delpiazzo, Carlos E., A la búsqueda del equilibrio entre privacidad y acceso, p. 2 [visible en internet: http://www.fder.edu.uy/contenido/pdf/9jornadas_idi.pdf, consultado el 8 de enero del 2013].

${ }^{12}$ Canales Gil, Álvaro, La protección de datos personales como derecho fundamental en Anuario Derecho Informático 4 (2004), pp. 264 s.

${ }_{13}$ Rajevic Mosler, Enrique, cit. (n. 1), pp. $4 \mathrm{~s}$.

${ }^{14}$ Sanz Salguero, Francisco Javier, Solicitud de acceso a la información y tutela de los datos personales de un tercero, en Revista de Derecho de la Pontificia Universidad Católica de Valparaíso 41 (2013) 2, p. 461.

${ }^{15}$ Recordemos que de todas las iniciativas legislativas vinculadas a la protección de la vida privada, la intimidad y los datos personales, hasta el momento solo tres han obtenido carácter normativo: la Ley No 19.423, que modificó el Código Penal, introduciendo tipos penales destinados a proteger la vida privada de las personas; la Ley No 19.628, denominada "Sobre protección de la vida privada", subtitulada "Protección de datos de carácter personal"; y la Ley No 19.812, modificatoria de la 
"Ley sobre protección de la vida privada", además de "Ley sobre protección de datos de carácter personal'), si bien es cierto en sus orígenes pretendía resguardar el derecho a la vida privada, al final terminó enfocada a la tutela de los datos personales.

3. Naturaleza jurídica del derecho de acceso a la información pública dentro del marco de la Constitución Politica: puntos de vista a tener en cuenta en su identificación

A diferencia de lo que ocurre con los datos personales, cuya protección se encuentra claramente reconocida a nivel constitucional en el artículo 19 No $4^{\circ} \mathrm{CPol}$. (y en el plano normativo en la Ley No 19.628), determinar el carácter iusfundamental o no del acceso a la información exige un trabajo más intenso, dadas las posturas que la doctrina y la jurisprudencia plantean sobre esta figura en particular. A continuación entonces, con el objeto de adoptar una posición en esta materia, expondremos los diversos puntos de vista formulados en cuanto a la naturaleza jurídica del derecho de acceso a la información pública dentro del marco de la Carta de Derechos, comenzando por la visión contenida en la jurisprudencia del Consejo para la Transparencia, pasando por el punto de vista que sobre el tema formula el Tribunal Constitucional, concluyendo con el estudio de la posición doctrinal que considera el acceso a la información como un derecho de naturaleza iusfundamental adscripto al texto de la Carta Política.

\section{Naturaleza jurídica del derecho de acceso a la información pública} dentro del marco de la Carta Política: punto de vista del Consejo para la Transparencia

Siguiendo la línea de estudio propuesta, una primera visión que debemos tener en cuenta es la planteada en la jurisprudencia del Consejo para la Transparencia. Para este organismo, el derecho de acceso a la información estaría consagrado en el nuevo artículo 8 de la Carta Fundamental ${ }^{16}$, norma que hace parte del Capítulo I de las "Bases de la Institucionalidad"17

Ley No 19.628. Véase: Anguita Ramírez, Pedro, La protección de datos personales y el derecho a la vida privada, régimen jurídico, jurisprudencia y derecho comparado (Santiago, Editorial Jurídica de Chile, 2007), p. 229.

${ }^{16}$ Norma que surgió a partir de la modificación a la CPol. ocurrida en el año 2005 (Ley No 20.050).

17 En palabras del profesor Hugo Tórtora Aravena, se entiende por "Bases de la Institucionalidad" el conjunto de principios que sirven de soporte o cimiento conforme al cual se organiza la sociedad. El mismo autor, nos recuerda que éste capítulo fija la estructura esencial que inspirará el resto de la normativa constitucional, estableciendo entre otros tópicos las normas básicas en relación con las personas, con la forma del Estado y del Gobierno, con los deberes del Estado, y con regulación 
y que se identifica, por ende, como un principio de rango constitucional. Por ejemplo, el Consejo al proponer la aplicación del artículo $5^{\circ}$ inciso $2^{\circ}$ de la "Ley de transparencia" (artículo que le otorga carácter público a la información elaborada con presupuesto público y toda otra información que obre en poder de los órganos de la Administración, cualquiera sea su formato o so porte, entre otros casos) dentro del ámbito de los correos electrónicos intercambiados por funcionarios públicos ${ }^{18}$, ha considerado que la extensión del mencionado artículo $5^{\circ}$ inciso $2^{\circ}$ debe analizarse teniendo a la vista dos de los principios del derecho de acceso a la información que reconoce el artículo 11 de la misma ley: el principio de relevancia (conforme al cual se presume relevante toda información que posean los órganos de la Administración del Estado, cualquiera sea su formato, soporte, fecha de creación, origen, clasificación o procesamien$\operatorname{tos}^{19}$ ), y el principio de apertura o transparencia (de acuerdo al cual "toda la información en poder de los órganos de la Administración del Estado se presume pública, a menos que esté sujeta a las excepciones señaladas" $\left.{ }^{20}\right)$. En este contexto, para el Consejo combinando estos preceptos resulta claro que el legislador ha extendido la publicidad de la información más allá del tenor del artículo $8^{\circ}$ de la Constitución, que sólo declara públicos "los actos y resoluciones de los órganos del Estado, así como sus fundamentos y los procedimientos que utilicen" 21 .

5. Naturaleza jurídica del derecho de acceso a la información pública dentro del marco de la Carta Política: punto de vista del Tribunal Constitucional En segundo lugar, desde la óptica del Tribunal Constitucional y en

del ejercicio de la Soberanía Nacional. Véase: Tótora Aravena, Hugo, Apuntes derecho constitucional. [visible en Internet: http://constitucionalchile.blogspot. com/2010/07/02-bases-de-la-institucionalidad.html, consultado el 24 de abril del 2014].

${ }^{18}$ Decisión de Amparo No C1101-11, del 16 de marzo de 2012.

${ }^{19}$ Artículo 11 letra a) de la Ley de transparencia.

${ }^{20}$ Artículo 11 letra c) de la Ley de transparencia.

${ }^{21}$ Este último razonamiento, va en sintonía con lo expresado por los organismos que patrocinan el derecho de acceso en Chile, bajo cuya opinión el limitar los alcances del acceso a la información pública a que tal información exista en un procedimiento administrativo o bien forme parte de los fundamentos de actos administrativos, implicaría poner en riesgo el principio de la transparencia máxima, situación que según esta perspectiva pondría al país en un retroceso, llevándolo de paso a infringir las leyes internacionales y los compromisos políticos que el Estado ha asumido en el concierto internacional. Véase: Alianza Regional pOR la Libre EXPRESIÓN E Información, Saber Más VI: Informe Regional sobre acceso a la información pública $y$ las instancias de control y apelación (Montevideo, Alianza Regional por la Libre Expresión e información, 2014), pp. 36 s. 
contraposición a lo expresado por el Consejo para la Transparencia, el artículo $8^{\circ}$ de la Carta Fundamental no consagra un derecho de acceso a la información, derecho que "más bien, se encuentra reconocido i mplic ita me n te en el artículo 19 numeral 12 de la Constitución"22 (el destacado es nuestro), específicamente en la parte de la norma que establece como derecho "La libertad de emitir opinión y la de informar". En efecto, para este Tribunal el derecho de acceso a la información pública se encuentra reconocido en la Constitución de forma im plícit a ${ }^{23}$, dado el carácter de república democrática que posee Chile y por virtud del cual "las autoridades públicas responden a la sociedad; por lo tanto tiene a disposición de los ciudadanos los actos y da cuenta de ellos" 24 .

En sintonía con la postura anterior, la autoridad constitucional agrega que el derecho de acceder a las informaciones que obran en poder de los órganos del Estado, forma parte de la libertad de expresión, figura ésta última que (en palabras del Tribunal) se encuentra consagrada en el mencionado artículo 19 numeral 12 de la Carta Política ${ }^{25}$. Ésta opinión concuerda con lo expresado por la Corte de Apelaciones de Santiago, autoridad según la cual en la garantía del precitado artículo 19 numeral 12, relativa a la libertad de información, "debe entenderse comprendido el derecho a recibir información, ya que como se ha sostenido por diversos autores, de nada serviría que se aseguraran la libertad de emitir opinión y la de información si no se reconoce que los destinatarios tienen, a su vez, el legitimo derecho a recibir una información oportuna, veraz y completa" 26 .

Continuando con la exposición, para el Tribunal debe tenerse en cuenta el vínculo existente entre el derecho de acceso a la información pública, en relación con el artículo $5^{\circ}$ inciso $2^{\circ}$ de la Constitución, y el artículo 13.1 de la Convención Americana de Derechos Humanos o CADH (norma ésta última que reconoce la libertad de buscar y recibir información) ${ }^{27}$. Finalmente (en concordancia con la opinión de la autoridad constitu-

22 Sentencia del Tribunal Constitucional, Rol No 2246-2012 del 31 de enero de 2013, Considerando 22\%.

${ }^{23}$ Desde la órbita de lo lingüístico, el Diccionario de la lengua española DRAE estima que algo es "implícito", cuando esta "incluido en otra cosa sin que esta lo exprese".

${ }^{24}$ Sentencia del Tribunal Constitucional, Rol No 634/2006, de 9 de agosto de 2007, Considerando 9º

${ }^{25}$ Sentencia del Tribunal Constitucional, Rol No 634/2006, de 9 de agosto de 2007, Considerando 90.

${ }^{26}$ Corte de Apelaciones de Santiago, sentencia de Roles acumulados No 5527 2001 y No 5728-2001, Considerando $9^{\circ}$.

${ }_{27}$ Sentencia del Tribunal Constitucional, Rol No 634/2006, de 9 de agosto de 2007, foja 745 . 
cional conforme a la cual, el derecho a acceder a las informaciones que obran en poder de los órganos del Estado forma parte de la libertad de expresión), en cuanto al rol del artículo $8^{\circ}$ de la Carta Fundamental, para el Tribunal Constitucional el ejercicio de la libertad de información se ve reafirmado por el bien jurídico de la transparencia, bien jurídico que tiene la calidad de base de la institucionalidad, y que precisamente se encuentra establecido en el numeral $2^{\circ}$ de la citada norma. Recordemos que el precepto constitucional, estipula que "Son públicos los actos y resoluciones de los órganos del Estado, así como sus fundamentos y los procedimientos que utilicen", salvo las excepciones señaladas en el mismo artículo ${ }^{28}$.

\section{Naturaleza jurídica del derecho de acceso a la información pública den-} tro del marco de la Carta Política: punto de vista de la tesis de la adscripción

En tercer lugar y desde la óptica de los derechos fundamentales, un sector de la doctrina considera que el acceso a la información es un derecho de naturaleza iusfundamental ad s crip to ${ }^{29}$ al texto de la Carta de Derechos, y no un derecho de carácter implícito (tal como lo ha planteado la jurisprudencia del Tribunal Constitucional ${ }^{30}$. Los argumentos que sostienen esta visión del acceso a la información como derecho fundamental de naturaleza adscripta a la Constitución, se sintetizan asî́ ${ }^{31}$ :

a) En primer lugar, quienes promueven ésta visión identifican la adscripción de normas iusfundamentales a un texto constitucional como una actividad básicamente argumentativa, ya sea en sede doctrinal o jurisprudencial pero, ciertamente, normativa y no empírica (como la mera constatación de adscripciones por determinados operadores jurídicos).

${ }^{28}$ Sentencia del Tribunal Constitucional, Rol No 634/2006, de 9 de agosto de 2007, foja 746 .

${ }^{29}$ Desde la órbita de lo lingüístico, el Diccionario de la lengua española DRAE establece que la "adscripción" (término que proviene del latín adscript o, - nis) es la "acción y efecto de adscribir", definiendo la acción de "adscribir" como "inscribir, contar entre lo que corresponde a alguien o algo".

30 Apoyan la visión de la adscripción iusfundamental del derecho de acceso a la información, académicos como Gonzalo García Palominos y Pablo Contreras V.

31 Es pertinente subrayar que quienes apoyan la visión de la adscripción iusfundamental del derecho de acceso a la información, estiman que la noción de derecho fundamental debe ir más allá de la esfera puramente normativa, abarcando también la lucha histórica que garantiza, especifica y universaliza el más amplio reconocimiento de la dignidad humana. Esta reflexión, surge apropósito de la necesidad por conocer la verdad histórica (dentro del marco de lo acontecido durante los años 1973 a 1990 en Chile) y estimular la protección de los derechos humanos (a partir del retorno a la democracia). Véase: García P. Gonzalo y Contreras V. Pablo, Derecho de acceso a la información en Chile: Nueva regulación e implicancias para el sector de la defensa nacional, en Revista de Estudios Constitucionales 7 (2009) 1, p. 139. 
Bajo ésta noción, más allá de las normas iusfundamentales estatuidas, las normas adscriptas de derecho fundamental son aquellas que, dada una determinada relación de fundamentación (iusfundamentalmente correcta), se adscriben a normas iusfundamentales directamente estatuidas ${ }^{32}$.

b) Concretamente, sobre la condición de derecho fundamental del derecho de acceso a la información, el argumento más relevante que debe tenerse en cuenta desde la perspectiva de la adscripción lo constituye la interpretación que la Comisión Interamericana de Derechos Humanos (en adelante CIDH) realiza de la CADH (también conocida como "Pacto de San José"), interpretación según la cual el derecho de acceso a la información es un derecho fundamental recogido por la $\mathrm{CADH}$ en su artículo 13 , norma que reconoce la libertad de buscar y recibir información (y que hacen parte de la libertad de pensamiento y de expresión) ${ }^{33}$. Precisamente, esta consideración fue confirmada por la Corte Interamericana de Derechos Humanos (en adelante Corte IDH) en la sentencia proferida el 19 de septiembre de 2006 en el Caso Claude Reyes y otros contra Chile ${ }^{34}$.

En este orden de ideas ¿Cuál es el contexto normativo interno en el que surge la citada sentencia? En concordancia con el anterior interrogante ¿Cuál es la consideración principal expresada en la sentencia, en lo que concierne al acceso a la información? En efecto, el derecho de acceso a la información pública vino a ser reconocido en Chile por la Ley No 19.653 de 1999 sobre probidad administrativa (estatuto que además de consagrar como regla general la publicidad de los actos administrativos, r e s t r i n g i ó la reserva a un listado basado en cuatro causales que podía ser desarrollado por vía reglamentaria y admitiendo una impugnación en sede judicial $\left.{ }^{35}\right)^{36}$. Sin embargo, un giro al respecto se presentó en septiembre del 2006 con la Sentencia de la Corte IDH en el caso Claude Reyes, la cual declaró que el sistema de acceso a la información chileno infringía el artículo 13 de la $\mathrm{CADH}$, pues éste garantizaba "el derecho que tiene toda persona a solicitar el acceso a la información bajo el control del Estado" (párrafo 77). De tal manera que, para la Corte, con base en el artículo 5 de la CPol. chilena (norma

32 Ibíd., p. 141.

33 Puntualmente, el numeral $1^{\circ}$ artículo 13 de la Convención Americana de Derechos Humanos (que consagra la libertad de pensamiento y opinión) reza "Toda persona tiene derecho a la libertad de pensamiento y de expresión. Este derecho comprende la libertad de buscar, recibir y difundir informaciones e ideas de toda indole, sin consideración de fronteras, ya sea oralmente, por escrito o en forma impresa o artística, o por cualquier otro procedimiento de su elección".

${ }^{34}$ García P. Gonzalo y Contreras V. Pablo, cit. (n. 31), p. 141.

35 Artículo 14, Ley No 19.653 de 1999.

${ }^{36}$ Rajevic Mosler, Enrique, cit. (n. 1), pp. 146 s. 
que exige a los órganos del Estado respetar y promover los derechos esenciales que emanan de la naturaleza humana garantizados "por los tratados internacionales ratificados por Chile y que se encuentren vigentes", incluido el "Pacto de San José") éste derecho de acceso se habría incorporado en la Carta Fundamental ${ }^{37}$.

c) Conforme a lo explicado entonces, desde la esfera de los defensores de la adscripción, el derecho de acceso a la información es una norma iusfundamental adscripta al artículo 19 numeral 12 y numeral 14, al artículo $8^{\circ}$ y al artículo $5^{\circ}$ inciso $2^{\circ}$ de la Constitución que reenvía el ordenamiento constitucional a su complementariedad con el artículo 13 de la $\mathrm{CADH}^{38}$. Según esta visión, todas estas últimas son normas iusfundamentales directamente estatuidas y que permiten la fundamentación de una norma adscrita a ellas. Sin embargo, la cuestión sobre la posición constitucional de los derechos reconocidos por los tratados internacionales, ha sido un tema objeto de controversia en Chile en el último tiempo, tal como lo recuerda Aldunate Lizana ${ }^{39}$. En este sentido, observamos dos puntos de vista al respecto: por un lado, un sector de la doctrina considera que a partir de la reforma introducida al señalado artículo $5 \mathrm{CPol} .{ }^{40}$, se había elevado a rango constitucional los tratados internacionales (ratificados y vigentes) en materia de derechos humanos (anotándose incluso una variante a esta opinión según la cual, lo que se había incorporado a la Constitución y a su jerarquía no eran los tratados, sino los derechos contenidos en ellos), opinión adoptada por autores como Pinochet Elorza ${ }^{41}$, Jara Castro ${ }^{42}$ y Medina Quiroga ${ }^{43}$. Al contrario, otro sector estima inadmisible la tesis de elevación de jerarquía normativa de los tratados, a partir de la modificación del citado artículo 5, siendo de esta opinión autores como Saenger Gianoni ${ }^{44}$.

\footnotetext{
37 Ibíd., p. 147.

38 García P. Gonzalo y Contreras V. Pablo, cit. (n. 31), p. 141.

39 Aldunate Lizana, Eduardo, cit. (n. 5), pp. 338-345.

${ }^{40}$ Modificación introducida por la Ley No 18.825 de 1989 que reformó la Constitución.

${ }^{41}$ Pinochet Elorza, Cesar, Eficacia de la elevación a rango constitucional de los tratados internacionales sobre derechos humanos, en Cuadernos de análisis jurídico (1990) 13, p. 11.

42 JARA CASTRO, Eduardo, La modificación al inciso segundo del artículo 5 de la Constitución de 1980 y la Ley 18.857, en Cuadernos de análisis jurídico (1990) 14, p. 18.

${ }^{43}$ Medina Quiroga, Cecilia, Constitución, tratados y derechos esenciales (Santiago, Corporación Nacional de Reparación y reconciliación, 1994), p. 49.

${ }^{44}$ Este autor, a partir de la esfera constitucional, reconoce la importancia de los tratados internacionales, en especial en lo concerniente al ámbito de los derechos humanos. Sin embargo, incluye a estos tratados en el marco del ordenamiento
} 
Esta última idea es compartida incluso por el Tribunal Constitucional en su jurisprudencia la cual, en síntesis, estima inadmisible el otorgamiento de jerarquía constitucional a los tratados internacionales sobre derechos humanos (con base en la modificación del mencionado artículo 5o), ya que esta pretensión chocaría con el sistema mixto de control de constitucionalidad de la ley entregado al Tribunal Constitucional y a la Corte Suprema (control tanto preventivo como represivo), sistema mixto que no sería operable si los tratados internacionales sobre derechos humanos tuvieran rango constitucional per se, con el agravante de la posible afectación al mecanismo de reforma de la Carta Fundamental contenido en su Capítulo XIV, mecanismo que posee un carácter particularmente rígido ${ }^{45}$.

d) Continuando con la exposición, para el sector doctrinal que respalda la adscripción iusfundamental del derecho de acceso a la información, la teoría general de los derechos fundamentales permite fundar el reconocimiento de normas iusfundamentales a través de la praxis argumentativa de los operadores jurídicos ${ }^{46}$, opinión según la cual nos encontramos frente a un derecho fundamental que requiere las obvias especificaciones interpretativas que lo reafirman en su condición y que ni el Tribunal Constitucional ni la Corte Internacional podría desconocer a partir de estos textos. Entonces, según esta concepción doctrinal, la tesis de los derechos "implícitos" impulsada por el Tribunal Constitucional, nos haría enfrentar problemas cognoscitivos que surgen con una tesis que incluye derechos no expresamente recogidos por la Carta Política, problemas como por ejemplo: cuál es su contenido y cómo debemos entender estos derechos implícitos,

jurídico chileno dentro de la misma categoría de las leyes comunes. El mismo autor, concluye que la vigencia de los tratados supranacionales en materia de derechos humanos, depende de su incorporación formal a la Carta Política. Véase: SAENGer Gianoni, Fernando, Consideraciones para estimar que los tratados en materia de derechos humanos no forman parte de la Constitución, en Revista Chilena de Derecho 20 (1993) 2-3, p. 667.

45 Sentencia del Tribunal Constitucional, Rol No 346/2002, de 8 de abril de 2002, Considerando 68.

46 El sector doctrinal que avala la adscripción iusfundamental del derecho de acceso a la información, respalda su opinión teniendo en cuenta la "teoría jurídica general de los derechos fundamentales de la ley fundamental" formulada por Robert Alexy. El autor, distingue ésta de otras teorías referentes a los derechos fundamentales, como por ejemplo las teorías históricas (que explican el surgimiento de los derechos fundamentales), las filosóficas (que se ocupan de su fundamentación) y las sociológicas (las cuales estudian la función de los derechos fundamentales en el sistema social), reconociendo el aporte que todas las disciplinas en la esfera de las ciencias sociales pueden proporcionar a la problemática de los citados derechos. Véase: Alexy, Robert, Teoría general de los derechos fundamentales (traducción de Ernesto Garzón Valdés, Madrid, Centro de Estudios Constitucionales, 1993), p. 27. 
cuál es el método para su reconocimiento, y cuál es su estatus y jerarquía normativa si los comparamos con el resto de los derechos establecidos en normas iusfundamentales ${ }^{47}$.

e) Finalmente, el sector de la doctrina que avala la adscripción iusfundamental del acceso a la información considera que este derecho, en tanto derecho fundamental, se entiende bajo la estructura de principio ${ }^{48}$. Es pertinente observar, que la noción de principio utilizada en éste contexto, es la planteada por Robert Alexy ${ }^{49}$, autor según el cual "los principios son normas que ordenan que algo sea realizado en la mayor medida posible, dentro de las posibilidades jurídicas y reales existentes", otorgándole a estos el carácter de "mandatos de optimización (entendido este concepto de mandato en un sentido amplio, que abarca también permisiones y prohibiciones), que están caracterizados por el hecho de que pueden ser cumplidos en diferente grado y que la medida debida de su cumplimiento no sólo depende de las posibilidades reales sino también de las jurídicas".

La adscripción de la norma, para este derecho de acceso a la información, se realizaría en conexión con cuatro factores: en primer lugar, la dimensión objetiva de la libertad de expresión; en segundo término, el derecho de petición individual sobre asuntos de interés público; en tercer lugar, la obligación de promoción y tutela del Estado en materia de derechos fundamentales y, en cuarto término, la conexión de éste derecho con la generación de una opinión pública libre y el desarrollo del sistema democrático deliberativo (factor éste último que estaría reconocido por la Corte IDH $\left.{ }^{50}\right)^{51}$. Entonces, por virtud de las normas iusfundamentales señaladas, el derecho de acceso a la información se traduciría en un derecho prestacional, en la generación de una obligación positiva del Estado de promover, permitir y garantizar el acceso a información pública, con las salvedades indicadas en el artículo $8^{\circ}$ de la Carta Política.

\section{Acceso a la información pública y su carácter de derecho fundamental}

${ }^{47}$ García P. Gonzalo y Contreras V. Pablo, cit. (n. 31), p. 142.

${ }^{48}$ Ibíd., p. 142.

49 Alexy, Robert, cit. (n. 46), p. 86.

50 Puntualmente, la CIDH dentro del caso Claude Reyes recuerda que la Asamblea General de la OEA en diversas resoluciones ha considerado "que el acceso a la información pública es un requisito indispensable para el funcionamiento mismo de la democracia, una mayor transparencia y una buena gestión pública, y que en un sistema democrático representativo y participativo, la ciudadanía ejerce sus derechos constitucionales, a través de una amplia libertad de expresión y de un libre acceso a la información". Véase: Corte InTERAmericana de Derechos Humanos, Caso Claude Reyes y otros contra Chile, Sentencia de 19 de septiembre de 2006, párrafo 84.

${ }^{51}$ García P. Gonzalo y Contreras V. Pablo, cit. (n. 31), p. 143. 
Partiendo de la base de que no habría duda del reconocimiento constitucional del derecho de acceso a la información pública (ya sea que éste reconocimiento tenga un carácter jurisprudencial o doctrinal ${ }^{52}$ ), el siguiente paso en el desarrollo de la presente investigación exige adoptar una posición en cuanto al carácter iusfundamental o de principio constitucional del citado derecho, exigencia que implica matizar el contenido de las tres visiones previamente estudiadas, puntos de vista que abarcan diversos ámbitos de la juridicidad chilena, incluyendo esferas como la normativa, la jurisprudencial y la doctrinal. A continuación, desarrollaremos la labor de análisis propuesta.

a) En primer lugar, interpretando la visión del órgano rector de la transparencia, el acceso a la información es un principio de rango constitucional, ya que se encuentra reconocido en el nuevo artículo 8 de la Carta Fundamental, norma que hace parte del Capítulo I de las "Bases de la Institucionalidad" (es decir, del conjunto de principios que sirven de soporte o cimiento conforme al cual se organiza la sociedad ${ }^{53}$ ). Entonces, bajo la perspectiva del Consejo para Transparencia, el acceso a la información no tendría el carácter de derecho fundamental, siendo su naturaleza la de un principio constitucional.

b) En segundo lugar, según la visión de la máxima autoridad constitucional, el acceso a la información sería un derecho reconocido i m p lí c i ta men te en el artículo 19 numeral 12 de la CPol., específicamente en la parte de la norma que establece como derecho "La libertad de emitir opinión y la de informar". Podemos identificar, dentro de la perspectiva del Tribunal Constitucional, los siguientes elementos a efecto de establecer cuál es la naturaleza jurídica (derecho fundamental o principio constitucional) del acceso a la información: i) desde la órbita de lo lingüístico, el Diccionario de la Real Academia Española DRAE estima que algo es "implícito" cuando esta "incluido en otra cosa sin que esta lo exprese". Con base en la anterior definición, si nos atenemos al ámbito estrictamente gramatical, en opinión del Tribunal el acceso a la información haría parte de un derecho fundamental (concretamente, "La libertad de emitir opinión y la de informar"), aunque este derecho fundamental no lo declare o manifieste (o, en otras palabras, no lo exprese).

52 Este reconocimiento se deduce del material estudiado en esta parte de la investigación. Por ejemplo, para la jurisprudencia del Tribunal Constitucional, el reconocimiento constitucional del derecho de acceso a la información pública "no merece duda a la doctrina de los iuspublicistas y tampoco a la jurisprudencia". Sentencia del Tribunal Constitucional, Rol No 634/2006, de 9 de agosto de 2007, Considerando $9^{\circ}$.

53 Tórtora Aravena, Hugo, cit. (n. 17). 
ii) En cuanto a la estructura de la Carta Política, a pesar del problema que plantea la nomenclatura de los derechos en el ordenamiento constitucional chileno ${ }^{54}$, no hay duda respecto al carácter de derecho fundamental que posee "La libertad de emitir opinión y la de informar" al estar consagrado expresamente en el artículo 19 de la Constitución ${ }^{55}$. Sin embargo esta norma, como ya lo señalamos, establece un catálogo no taxativo de derechos fundamentales, admitiéndose en consecuencia la posibilidad de que existan este tipo de derechos (en el sentido de esenciales, inherentes a la persona) no consagrados en la CPol. ${ }^{56}$. Hasta este punto entonces, podemos identificar al acceso a la información pública como una figura que, a pesar de no serlo expresamente, si hace parte de un derecho fundamental constitucional como lo es "La libertad de emitir opinión y la de informar".

Aplicando los factores anteriormente descritos, para la jurisprudencia del Tribunal Constitucional no podría catalogarse el acceso a la información como un derecho fundamental constitucional, es decir, no se identificaría con el concepto de derecho fundamental en su aspecto formal. Pero entonces, de acuerdo a esta afirmación ¿para el Tribunal el acceso a la información no es un derecho fundamental? ¿Será entonces acaso un principio constitucional? La jurisprudencia más reciente de la máxima autoridad constitucional ${ }^{57}$, admite que el de recho al acceso a la información se encuentra im plícitamente reconocido dentro de una norma constitucional (artículo 19 numeral 12), norma que consagra expresamente un derecho fundamental ("La libertad de emitir opinión y la de informar"). Bajo esta premisa, el acceso a la información (y más exactamente, el derecho de acceso a la información) debe entenderse (bajo la visión del Tribunal Constitucional) como un derecho fundamental (aunque, insistimos, no identificado con el concepto de derecho fundamental en su aspecto formal)

iii) En resumen, la anterior conclusión según la cual para el Tribunal

54 En relación a la sistemática de los derechos constitucionales, la doctrina reconoce la presencia de un problema en la nomenclatura especialmente fuerte en el ordenamiento constitucional interno. Lo anterior, teniendo en cuenta que la Constitución es particularmente generosa de los derechos, sin que pueda, en todos los casos, estimarse que se trata simplemente de denominaciones diversas para un mismo objeto. Véase: Aldunate Lizana, Eduardo, cit. (n. 5), p. 335.

55 Esta afirmación en particular, es apoyada a nivel legal por normas como la Ley No 19.733 sobre libertades de opinión e información y ejercicio del periodismo, estatuto que en su artículo $1^{\circ}$ declara que "la libertad de emitir opinión y la de informar, sin censura previa, constituyen un derecho fundamental de todas las personas".

${ }^{56}$ Aldunate Lizana, Eduardo, cit. (n. 5), p. 345.

57 Puntualmente, la Sentencia Rol No 2246-2012 del 31 de enero de 2013, previamente estudiada. 
Constitucional el acceso a la información debe identificarse como un derecho fundamental, se sustenta en los siguientes argumentos: en primer lugar, la consideración gramatical de la expresión "implícito", y el ámbito (desde el punto de vista de la estructura de la Carta Política) donde el derecho de acceso se encuentra reconocido implícitamente como un derecho fundamental. En segundo lugar, la opinión mayoritaria de la doctrina que estima el catálogo del artículo 19 de la Constitución (catálogo en el que son consagrados los derechos de carácter fundamental) como no taxativo, admitiéndose así la posibilidad de que existan derechos fundamentales (en el sentido de esenciales, inherentes a la persona) no consagrados en la Carta Política. Y, en tercer lugar, íntimamente ligado al argumento anterior y desde la perspectiva de los derechos de creación o reconocimiento jurisprudencial, observamos el hecho de que en la práctica jurisprudencial el Tribunal ya ha aceptado la existencia de derechos fundamentales distintos a los expresamente reconocidos en la Constitución ${ }^{58}$. Como ejemplos de esta aceptación tenemos, en primer término ${ }^{59}$, el otorgamiento del carácter de derecho fundamental a la carrera funcionaria de un servidor público, transformando así una norma o principio organizador de la administración pública en un derecho subjetivo con carácter fundamental ${ }^{60}$; en segundo término ${ }^{61}$, tenemos la afirmación por parte del Tribunal de la existencia de un derecho de acceso a la información pública reconocido por la Carta Política "aunque lo sea de forma implícita", fallo en el que la máxima instancia constitucional sostiene que la referencia a la expresión "derechos" contenida en el artículo $8^{\circ}$ de la Constitución, no sólo debe entenderse comprensiva de derechos subjetivos, sino también de intereses legítimos ${ }^{62}$;

${ }^{58}$ Ya a mediados de la década de los noventa, el Tribunal Constitucional expreso que "tanto la doctrina como nuestra Constitución Política reconocen la existencia de derechos aunque no estén consagrados en el texto constitucional". Sentencia del Tribunal Constitucional, Rol No 226/1995, de 30 de octubre de 1995, Considerando $25^{\circ}$.

59 Sentencia del Tribunal Constitucional, Rol No 239/1996, de 16 de julio de 1996, Considerandos $8^{\circ}$ y $9^{\circ}$.

${ }^{60}$ Para Aldunate Lizana, esta calificación de un principio organizativo como derecho fundamental, plantea problemas en su función respecto de la actividad legislativa y dificultades a nivel dogmático. Véase: Aldunate Lizana, Eduardo, cit. (n. 5), pp. 345 s.

${ }^{61}$ Sentencia Rol No 634/2006, de 9 de agosto de 2007, Considerando $10^{\circ}$.

${ }^{62}$ Esta afirmación del Tribunal, contenida en los considerandos $21^{\circ}$ y $23^{\circ}$ de la Sentencia Rol No 634/2006, es criticada por Aldunate Lizana. Para el autor, el pretender que la expresión "derechos" contenida en el artículo $8^{\circ}$ de la Constitución debe entenderse comprensiva de derechos subjetivos e intereses legítimos (tal como lo plantea el Tribunal Constitucional), abre la puerta a una serie de problemas tanto para la dogmática constitucional de los derechos fundamentales, como para 
y en tercer término, con base en su más reciente jurisprudencia, tenemos la afirmación por parte del Tribunal según la cual el derecho al acceso a la información se encuentra implícitamente reconocido dentro de un derecho fundamental constitucional (contenido en el artículo 19 numeral 12), norma que consagra expresamente el derecho a "La libertad de emitir opinión y la de informar"63.

c) En tercer lugar, desde la visión de quienes promueven la figura del acceso a la información como un derecho de naturaleza iusfundamental a d s c r i p to al texto de la Constitución, no habría duda respecto a la condición de derecho fundamental del acceso a la información. Esta posición doctrinal, tiene su núcleo en la interpretación que la $\mathrm{CIDH}$ efectúa de la $\mathrm{CADH}$, interpretación según la cual el derecho de acceso a la información es un derecho fundamental recogido por la CADH en su artículo 13, norma que reconoce la libertad de buscar y recibir información (y que hacen parte de la libertad de pensamiento y de expresión), consideración confirmada por la Corte IDH en la sentencia proferida el 19 de septiembre de 2006 en el caso Claude Reyes. En sintonía con lo anterior, quienes apoyan esta visión van más allá de la tesis de los derechos “implícitos” promovida por el Tribunal Constitucional, en atención a los problemas cognoscitivos que surgen con una tesis que incluye derechos no expresamente recogidos por la Carta Política.

d) En conclusión, y después de matizar el contenido de las tres visiones sobre la naturaleza (o, si se quiere, la jerarquía) del acceso a la información pública dentro del marco de la Carta Política ${ }^{64}$, pensamos que éste posee el carácter de derecho fundamental. En efecto, son varios los aspectos estudiados que sustentan nuestra posición: i) si bien es cierto la postura del Tribunal Constitucional es la de otorgarle al acceso a la información el estatus de derecho "implícito" (es decir incluido, aunque no sea invocado expresamente) en un derecho fundamental claramente constitucional (" $\mathrm{La}$ libertad de emitir opinión y la de informar"), y la tesis de la adscripción promueve a la figura del acceso a la información como un derecho de naturaleza iusfundamental adscripto al texto de la Constitución (adscripción al artículo 19 numeral 12 y numeral 14 , artículo $8^{\circ}$ y artículo 5 inciso $2^{\circ}$

la conducción de las dogmáticas especiales de las distintas ramas del derecho. Véase: Aldunate Lizana, Eduardo, cit. (n. 5), p. 347.

${ }^{63}$ Sentencia del Tribunal Constitucional, Rol No 2246-2012 del 31 de enero de 2013, Considerando 22。.

${ }^{64}$ Concretamente los puntos de vista del Consejo para la Transparencia, del Tribunal Constitucional y de la posición doctrinal que considera el acceso a la información como un derecho de naturaleza iusfundamental adscripto al texto de la Carta de Derechos. 
de la Carta Fundamental que reenvía el ordenamiento constitucional a su complementariedad con el artículo 13 de la $\mathrm{CADH}$ ), ambas posturas tienen un denominador común: conceden al acceso a la información la categoría de derecho fundamental. ii) En este contexto, y aunque se admitiera que el acceso a la información no es un derecho fundamental constitu cio nal (es decir, no identificado con el concepto de derecho fundamental en su aspecto formal), factores como el carácter no taxativo del artículo 19 de la Constitución, la aceptación de la existencia por parte del Tribunal Constitucional en su praxis jurisprudencial de derechos fundamentales distintos a los expresados en la Carta Política, y la existencia de la denominada "cláusula abierta" de los derechos fundamentales ${ }^{65}$, apoyan la idea de la presencia de un derecho fundamental al acceso a la información pública dentro de la esfera de la CPol. iii) A pesar de la discusión que persiste en la doctrina respecto a la posición constitucional de los derechos reconocidos por los tratados internacionales ${ }^{66}$, el ordenamiento jurídico chileno debe dar especial atención al carácter de derecho humano fundamental que, en el ámbito del derecho comparado, diversos instrumentos internacionales (verbi gratia la Ley Modelo Interamericana sobre Acceso a la Información Pública ${ }^{67}$ en conjunto con lo estipulado dentro del Caso Claude Reyes) conceden al acceso a la información. En este sentido, pensamos que la normativa interna no puede desconocer la progresiva aplicación por parte de los tribunales nacionales (y en particular, de los tribunales constitucionales), de los instrumentos internacionales de

65 Pese a que en el contexto latinoamericano Chile, Panamá, México y Cuba no reconocen expresamente en sus Constituciones a esta figura, la gran mayoría de los países en América Latina incluyen cláusulas abiertas dentro de sus cartas de derechos. Este fenómeno se presenta, en virtud de la progresiva aplicación por los tribunales nacionales y en particular por los tribunales constitucionales, de los instrumentos internacionales de derechos humanos a los efectos de su protección en el orden interno. Véase: BREWER-CARÍAS, Allan R., La aplicación de los tratados internacionales sobre derechos humanos en el orden interno. estudio de derecho constitucional comparado latinoamericano, documento preparado para el Curso sobre el sistema interamericano de protección de los derechos humanos y su repercusión en los órdenes jurídicos nacionales, organizado por la Corte Suprema de Justicia de la Nación de México y la Corte Interamericana de Derechos Humanos, celebrado en México D.F. el 16 de marzo de 2006, pp. 1 s.

${ }^{66}$ Controversia en la que, recordemos, un sector de la doctrina considera que a partir de la reforma introducida al artículo 5 CPol. se había elevado a rango constitucional los tratados internacionales (ratificados y vigentes) en materia de derechos humanos.

${ }^{67}$ Esta Ley modelo (o LMSAIP), fue objeto de aprobación en su cuarta versión mediante resolución AG/RES. 2607 (XL-O/10) del ocho de junio del 2010 en la Asamblea General de la OEA. 
derechos humanos a los efectos de su protección en el orden interno ${ }^{68} . i v$ ) Una ventaja que no podemos obviar, es que el reconocimiento del carácter de derecho fundamental al acceso a la información pública, en lo que respecta a la comprobada tensión entre este derecho y la protección de la información personal, constituye un paso necesario a efecto de superar las controversias que en la práctica jurisprudencial se han presentado entre las autoridades del poder judicial y el Consejo para la Transparencia, en asocio con las diferencias presentes en materia doctrinal sobre esta materia. La importancia de resolver esta discusión es clara, ya que involucra derechos de máxima jerarquía en el ordenamiento constitucional. $v$ ) Finalmente, y ya en cuanto al rol del artículo $8^{\circ}$ de la Carta Fundamental, el ejercicio de la libertad de información se ve "reafirmado" (término utilizado en la praxis jurisprudencial del Tribunal Constitucional) por el bien jurídico de la transparencia, bien jurídico que tiene la calidad de base de la institucionalidad, y que se encuentra establecido en el numeral $2^{\circ}$ de la citada norma. Recordemos que el precepto constitucional, estipula que "Son públicos los actos y resoluciones de los órganos del Estado, así como sus fundamentos y los procedimientos que utilicen", salvo las excepciones señaladas en el mismo artículo ${ }^{69}$.

\section{TRATAMIENTO DEL CONFLICTO ENTRE PERSONAS QUE ALEGAN DERECHOS FUNDAMENTALES}

Adoptada una posición respecto al carácter iusfundamental del acceso a la información pública, un siguiente paso implica establecer cómo resolver el conflicto entre personas que alegan derechos fundamentales. A fin de formular una respuesta al interrogante planteado, en esta parte del trabajo explicamos el concepto de colisión de derechos fundamentales y su distinción con figuras afines (pero no iguales) como la concurrencia de derechos, las fórmulas de solución propuestas para quienes aceptan la existencia de colisiones de derechos fundamentales, y la solución plateada por quienes rechazan esta posibilidad. A continuación, comentamos estas nociones.

\section{Concepto de colisión de derechos fundamentales y su distinción con la} concurrencia de derechos

En cuanto a su concepto, en palabras de Aldunate Lizana ${ }^{70}$ se habla de colisión o choque de derechos fundamentales cuando "el efecto jurídico de

68 Brewer-Carías, Allan R., cit. (n. 65), p. 1.

${ }^{69}$ Sentencia del Tribunal Constitucional, Rol No 634/2006, de 9 de agosto de 2007, foja 746.

${ }^{70}$ Aldunate Lizana, Eduardo, cit. (n. 5), p. 269. 
la protección iusfundamental alegada por un sujeto (titular del respectivo derecho), es incompatible con el efecto jurídico perseguido por otro sujeto a partir de un alegato de protección iusfundamental". El mismo autor opina que la colisión es correlativa a la idea de los derechos de terceros como límites a los derechos fundamentales.

A partir del concepto anterior y para tener clara la diferencia entre nociones, es necesario contrastar la colisión con la concurrencia de derechos. $\mathrm{Al}$ respecto, observamos que la figura de la colisión de derechos no debe confundirse con la denominada concurrencia de derechos. En efecto, en el primer caso (colisión) las posiciones de dos o más titulares de derechos fundamentales, se enfrentan o chocan de tal modo que el resultado concedido a uno (o unos) deviene en la negación o rechazo de la protección o amparo invocado por el otro (u otros). El segundo caso (concurrencia) implica el alegato por parte de un sujeto de una lesión, por un mismo acto o conducta, de dos o más derechos fundamentales. Una primera lectura de la consideración anterior, nos permite afirmar que la tensión entre el derecho de acceso a la información y la protección de los datos de carácter personal estaría incluida dentro de un marco de conflicto entre personas que alegan derechos fundamentales, y no dentro del ámbito de la concurrencia. A título de ejemplo, basta recordar la jurisprudencia del Consejo para la Transparencia, en donde observamos varias situaciones de conflicto ante la pretensión de acceso a datos personales (incluso de carácter sensible) incluidos en bases de datos de naturaleza pública, notándose una clara confrontación entre el derecho que tiene el ciudadano a requerir información en poder del Estado (por virtud de su titularidad al derecho de acceso a la información pública) respecto al derecho que tiene otro ciudadano a resguardar sus datos de carácter personal (consecuencia de ser titular del derecho a proteger su información personal) ${ }^{71}$.

\section{Soluciones propuestas por quienes aceptan la posibilidad de colisiones} de derechos fundamentales

Si partiéramos de la base de que es posible la colisión de derechos ${ }^{72}$, un

${ }^{71}$ Sobre el particular y para tener en cuenta SAnZ SALguero, Francisco Javier, Solicitud de acceso a la información y tutela de los datos personales de un tercero, en Revista de Derecho de la Pontificia Universidad Católica de Valparaiso 41 (2013) 2, pp. 489-500, en donde desarrollamos una síntesis de la jurisprudencia del Consejo para la Transparencia, desde el punto de vista de la relación entre el acceso a la información pública y la protección de la información personal.

${ }^{72} \mathrm{La}$ tesis que acepta la posibilidad de colisión de derechos, parte de la idea de que se puede producir una contradicción ente la protección otorgada a los derechos fundamentales de dos o más titulares, de tal modo que, en estos casos, uno debe 
siguiente paso exigiría identificar el criterio más adecuado para determinar la prevalencia de unos derechos por sobre otros. En este sentido, la doctrina propone tres fórmulas, a saber ${ }^{73}$ :

a) En primer lugar, tenemos la idea de un orden de prelación o jerarquía, determinable en abstracto, entre los diferentes derechos (tesis de la jerarquización de derechos). Respecto a la aplicación de esta alternativa de la jerarquización, Aldunate Lizana crítica el hecho de la inexistencia de criterios objetivos que permitan establecer una jerarquía entre derechos fundamentales, y la ausencia de herramientas que posibiliten la determinación del logro de la máxima protección de cada uno de los derechos en colisión.

b) En segundo lugar, tenemos la fórmula de la ponderación de derechos en el caso concreto, postura según la cual serán las circunstancias del caso las que aportarán los elementos determinantes de la preferencia que deba darse a un derecho por sobre otro, pero solo en ese caso puntual. Esta opinión se encuentra fuertemente ligada a lo planteado por Alexy ${ }^{74}$, autor que defiende la concepción de los derechos como principios, y que constituye una de las piedras angulares de la tesis de la adscripción iusfundamental del acceso a la información dentro del marco de la Constitución. Respecto a la posibilidad de aplicación de esta alternativa de la ponderación, se crítica la falta de criterios objetivos que permitan la ponderación en un caso concreto, y (similar a lo que ocurre con la tesis de la jerarquización) la ausencia de elementos que posibiliten la determinación del logro de la máxima protección de cada uno de los derechos en colisión.

c) En tercer lugar, y como una variante de la ponderación (y por ende, sujeta a las mismas críticas realizadas a esta figura), tenemos la idea de armonización o del equilibrio adecuado. Esta opinión no pretende la búsqueda de un criterio que permita primar a un derecho por sobre el otro, ni siquiera en el caso concreto (como ocurre con la tesis de la ponderación), enfocándose en la búsqueda de una solución que facilite la óptima protección de los derechos enfrentados ("óptima protección" que se identifica como una diferencia a la figura de la ponderación).

\section{Soluciones propuestas por quienes rechazan la posibilidad de colisiones} de derechos fundamentales

Teniendo presente las críticas formuladas a las tres anteriores posturas (jerarquización, ponderación y armonización o del equilibrio adecuado),

prevalecer, mientras que el otro queda desplazado. Véase: Martínez-Pujalte, Antonio-Luis, La garantía del contenido esencial de los derechos fundamentales, (Madrid, Centro de Estudios Constitucionales, 1997), p. 527.

73 Aldunate Lizana, Eduardo, cit. (n. 5), pp. 273-275.

${ }^{74}$ Alexy, Robert, cit. (n. 46), pp. 89 y ss. 
propuestas que buscan determinar la prevalencia de unos derechos por sobre otros, el establecer de manera definitiva si es admisible la colisión de derechos fundamentales nos exige revisar las dos posturas que niegan la posibilidad conceptual de la colisión de este tipo de derechos ${ }^{75}$. A este respecto, anotamos lo siguiente:

a) Una de las teorías que niega la posibilidad de existencia de colisión de derechos (en el ámbito conceptual), parte del supuesto según el cual reconociendo cada derecho fundamental un límite inmanente ${ }^{76}$ en los derechos de los demás, su contenido propio, por definición, ya se encuentra delimitado por los derechos de otros, de tal manera que ante una eventual colisión este choque solo se presentará, conceptualmente, cuando el titular de un derecho fundamental intenta amparar su actuar más allá de los límites inmanentes de ese derecho. Esta teoría, que es apoyada por autores como De Otto y Pardo ${ }^{77}$ y Martínez-Pujalde ${ }^{78}$, nos plantea un problema: "es la precisa determinación de ese límite para el caso concreto la que puede construir el conflicto o controversia que se suscite procesalmente, como un caso de colisión o choque de derechos"79.

b) Una segunda postura que niega la existencia de colisión de derechos en la esfera conceptual, resulta de considerar la noción de derecho fundamental y la función normativa que cumple. Esta opinión, se origina en la idea de que la mayoría de los derechos fundamentales implican la protección a ciertas esferas de libertad, a partir del reconocimiento a un derecho de libertad general ${ }^{80}$, gran parte de cuya extensión puede no estar provista

75 En esta parte, pasamos directamente a las posturas que niegan la posibilidad conceptual de colisión de los derechos fundamentales. Lo anterior, teniendo en cuenta los problemas que subyacen en la opinión antitética que acepta dicha posibilidad, y que están representados en la solución del conflicto entre particulares por vía de ponderación respecto los dos derechos fundamentales en tensión. Como ejemplo, se observa que la ponderación siempre tiene que llegar a una consecuencia difícilmente sustentable, a saber: respecto de su protección, en un caso, un derecho fundamental es, por así decirlo, "más fundamental" que otro. Véase: Aldunate Lizana, Eduardo, cit. (n. 5), pp. $278 \mathrm{s.}$

${ }^{76}$ Es pertinente recordar, que la expresión "inmanente" hace referencia a lo que es inherente a algo, o que va unido de un modo inseparable a su esencia, según lo establece el DRAE.

77 De Отto y Pardo, Ignacio, La regulación del ejercicio de los derechos y libertades en Martin-Retortillo, Lorenzo y De Otto y Pardo, Ignacio, Derechos fundamentales y Constitución (Madrid, Civitas, 1988) p. 135.

78 Martínez-Pujalte, Antonio-Luis, La garantía del contenido esencial de los derechos fundamentales (Madrid, Centro de Estudios Constitucionales, 1997), p. 133.

79 Aldunate Lizana, Eduardo, cit. (n. 5), pp. 276 s.

${ }^{80}$ Esta premisa, estima que subyace a los derechos o libertades fundamentales 
de tutela fundamental. Desde esta óptica, lo que el ciudadano ejerce es siempre su libertad (o bien los derechos configurados por el ordenamiento jurídico), pero no su derecho fundamental a una libertad protegida, de lo que se observa que la colisión no es de derechos fundamentales: el choque se presenta entre el ejercicio de la libertad de uno en desmedro de una libertad o derecho garantizado a otro. En este punto, surge un interrogante: ¿̨un derecho fundamental protege del ejercicio de ésta libertad? Esta pregunta puede ser resuelta examinando el ámbito de libertad jurídica amparado por el respectivo derecho, es decir, determinando la extensión en que puede ser ejercida una libertad sin encontrar una norma o un precepto que la limite. En últimas, como el límite o frontera la fija el derecho, la solución a la colisión de derechos debe ser buscada en el ordenamiento jurídico subconstitucional con base en la siguiente premisa: el enfrentamiento entre derechos fundamentales equivale a la existencia de dos esferas de libertad jurídica igualmente protegidas ${ }^{81}$.

\section{Solución al conflicto entre personas que alegan derechos fundamentales}

En conclusión, revisadas las dos tesis que niegan la posibilidad conceptual de la colisión de derechos fundamentales, subrayando la inconsistencia detectada en una de estas tesis (concretamente, aquella basada en la existencia de límites inmanentes en los derechos fundamentales), observamos que la solución más coherente que puede otorgarse al conflicto entre particulares que alegan derechos fundamentales, nos exige identificar este conflicto como la confrontación entre el ejercicio de la libertad de un individuo y la libertad o derecho de otro, choque que debe ser resuelto con base en las fronteras o límites trazados por el juez a partir del ordenamiento jurídico vigente. Conforme a lo expuesto, el choque o conflicto no puede ser solucionado a partir de un ejercicio de ponderación que pretenda establecer la supremacía de un derecho fundamental por sobre otro.

La anterior conclusión, si nos enfocamos al ámbito de la construcción de límites a partir del ordenamiento jurídico vigente (rol atribuido al juez), se ve reforzada por una de las consideraciones del sector de la doctrina que avala la adscripción iusfundamental del acceso

conquistados para cada país por el respectivo movimiento constitucionalista (y posteriormente, los incorporados a partir de opiniones doctrinarias o comparadas) un principio esencial del Estado de Derecho: el principio de libertad. En este contexto, los individuos gozan de una libertad general respecto del Estado, que éste último regula a través de la ley, en la medida en que los medios de control del derecho (en un Estado de Derecho) no son preventivos, sino que represivos. Véase: Aldunate LizANA, Eduardo, cit. (n. 5), p. 223.

${ }^{81}$ Ibíd., p. 278. 
a la información: dentro del trabajo de proyección de los límites de los derechos fundamentales (especialmente el del derecho de acceso a la información), la Corte IDH ha señalado que toda limitación de un derecho protegido por la $\mathrm{CADH}$ debe realizarse a través de la ley. En este sentido, los seguidores de la adscripción iusfundamental del acceso estiman que a la luz de ordenamiento jurídico interno, este presupuesto de limitación debe entenderse satisfecho, teniendo en cuenta dos factores: en primer término, que el artículo $8^{\circ}$ de la CPol. dota de competencia al legislador para restringir el derecho de acceso a la información en virtud de alguna de las causales que ahí se señalan y, en segundo término, que el legislador ha decidido regular el ejercicio de este derecho, así como la vigencia del principio de transparencia, en la "Ley de Transparencia"82. Es pertinente anotar entonces, que la postura de la adscripción iusfundamental del acceso refuerza la solución propuesta al conflicto entre particulares que alegan derechos fundamentales, y constituye uno de los elementos que se deben tener en cuenta a fin de superar la tensión identificada en la relación entre el derecho de acceso y la protección de la información personal.

\section{CONCLUSIONES}

1. El establecer el grado de equivalencia normativa entre la protección de los datos personales y el derecho de acceso a la información pública, implica tener en cuenta como punto de partida el reconocimiento de derecho fundamental constitucional (o derecho fundamental en su aspecto formal) atribuible a la vida privada, subrayando que los datos personales representan un tipo de información que precisamente hace parte de esta esfera de la vida privada, por lo que la protección de la esfera citada concierne y beneficia directamente a la información de naturaleza personal.

2. En relación a la identidad jurídica del derecho de acceso a la información pública dentro del marco de la Carta Política, partiendo de la base que para la doctrina (en opinión mayoritaria) el catálogo del artículo 19 de la Constitución no es taxativo, y recogiendo los puntos de vista que sobre el particular nos proporciona el Consejo para la Transparencia, el Tribunal Constitucional y la teoría que identifica el acceso a la información como un derecho de naturaleza iusfundamental adscripto al texto de la Carta de Derechos, en primer lugar pensamos que este derecho de acceso se encuentra reconocido por la Constitución, subrayando que el origen de este reconocimiento varía dependiendo de la visión que se adopte: según la opinión del Consejo para la Transparencia, estaría consagrado en el

${ }^{82}$ García P. Gonzalo y Contreras V. Pablo, cit. (n. 31), pp. 143 s. 
nuevo artículo 8 de la CPol.; según la opinión del Tribunal Constitucional, estaría reconocido implícitamente en el artículo 19 numeral 12 de la Carta de Derechos; según la teoría de la adscripción, estaría adscripto al artículo 19 numeral 12 y numeral 14 , al artículo $8^{\circ}$ y al artículo 5 inciso $2^{\circ}$ que reenvía el ordenamiento constitucional a su complementariedad con el artículo 13 de la CADH.

3. Establecido el reconocimiento constitucional del derecho de acceso a la información pública (ya sea que éste reconocimiento tenga un carácter jurisprudencial o doctrinal), matizando el contenido de los puntos de vista examinados (Consejo para la Transparencia, Tribunal Constitucional y teoría de la adscripción), una segunda deducción es que el acceso a la información pública, dentro del marco de la Carta Política, posee el carácter de derecho fundamental. A esta conclusión se llega aplicando, en co nju n to, los siguientes elementos:

a) El hecho que la jurisprudencia del Tribunal Constitucional y la tesis de la adscripción le reconocen al acceso a la información el estatus de derecho fundamental. En el primer caso (jurisprudencia del Tribunal Constitucional), el reconocimiento surge con el otorgamiento al acceso a la información del carácter de derecho "implícito" (es decir incluido, aunque no sea invocado expresamente) en un derecho fundamental claramente constitucional (puntualmente, "La libertad de emitir opinión y la de informar"). En el segundo caso (teoría de la adscripción), el reconocimiento se observa al considerar la figura del acceso como un derecho de naturaleza iusfundamental adscripto al texto de la Constitución, adscripción relativa al artículo 19 numeral 12 y numeral 14 , artículo $8^{\circ}$ y artículo 5 inciso $2^{\circ}$ de la Carta de Derechos .

b) El carácter no taxativo del artículo 19 de la Constitución, en asocio con la aceptación en la praxis jurisprudencial del Tribunal Constitucional de derechos fundamentales distintos a los expresados en la Carta Política.

c) La existencia de la denominada "cláusula abierta" de los derechos fundamentales.

d) El carácter de derecho humano fundamental que, en el ámbito del derecho comparado, diversos instrumentos internacionales (verbi gratia la Ley Modelo Interamericana sobre Acceso a la Información Pública en conjunto con lo estipulado dentro del Caso Claude Reyes) conceden al acceso a la información.

4. Confirmada la naturaleza jurídica de derecho constitucional fundamental atribuible al acceso a la información pública, la solución más adecuada ante el conflicto entre personas que alegan derechos fundamentales (como podría ocurrir cuando se invocan, de manera simultánea, el derecho de acceso y la tutela los datos personales) exige tener en cuenta, de acuerdo 
a lo examinado en la presente investigación, la imposibilidad conceptual de la colisión de derechos de carácter iusfundamental. La anterior afirmación, parte de la premisa según la cual frente a la controversia entre ciudadanos que alegan derechos fundamentales, este conflicto debe ser identificado como la confrontación entre el ejercicio de la libertad de un individuo y la libertad o derecho de otro, choque que se resuelve con base en las fronteras o límites trazados por el juez a partir del ordenamiento jurídico vigente, es decir, a partir del ordenamiento jurídico subconstitucional.

\section{BIBLIOGRAFÍA}

Aldunate Lizana, Eduardo, Derechos fundamentales, (Santiago, Legal Publishing, 2008).

AleXY, Robert, Teoría general de los derechos fundamentales (traducción de Ernesto Garzón Valdés, Madrid, Centro de Estudios Constitucionales, 1993).

Alianza Regional por la Libre Expresión e información, Saber Más VI: Informe Regional sobre acceso a la información pública y las instancias de control y apelación (Montevideo, Alianza Regional por la Libre Expresión e información, 2014).

Anguita Ramírez, Pedro, La protección de datos personales y el derecho a la vida privada, régimen jurídico, jurisprudencia y derecho comparado (Santiago, Editorial Jurídica de Chile, 2007).

CanAles GIL, Álvaro, La protección de datos personales como derecho fundamental en Anuario Derecho Informático 4 (2004).

Delpiazzo, Carlos E., A la búsqueda del equilibrio entre privacidad y acceso [visible en internet: http://www.fder.edu.uy/contenido/pdf/9jornadas idi.pdf].

García P. Gonzalo y Contreras V. Pablo, Derecho de acceso a la información en Chile: Nueva regulación e implicancias para el sector de la defensa nacional, en Revista de Estudios Constitucionales 7 (2009) 1.

Gomes Canotilho, José Joaquim, Direito Constitucional e Teoría da Constituição (5 edición, Almedina, Cohimbra, Portugal, 2002).

JaRA CASTRO, Eduardo, La modificación al inciso segundo del artículo 5 de la Constitución de 1980 y la Ley 18.857, en Cuadernos de análisis jurídico (1990) 14.

La aplicación de los tratados internacionales sobre derechos humanos en el orden interno. Estudio de derecho constitucional comparado latinoamericano, documento redactado por BREWER-CARíAs, Allan R. para el Curso sobre el sistema interamericano de protección de los derechos humanos y su repercusión en los órdenes jurídicos nacionales, organizado por la Corte Suprema de Justicia de la Nación de México y la Corte Interamericana de Derechos Humanos, celebrado en México D.F. el 16 de marzo de 2006.

Martin-Retortillo, Lorenzo y De Otto y Pardo, Ignacio, Derechos fundamentales y Constitución (Madrid, Civitas, 1988).

Martínez-Pujalte, Antonio-Luis, La garantía del contenido esencial de los derechos fundamentales, (Madrid, Centro de Estudios Constitucionales, 1997).

Medina Quiroga, Cecilia, Constitución, tratados y derechos esenciales (Santiago, Corporación Nacional de Reparación y reconciliación, 1994). 
PinOCHet ELORZA, Cesar, Eficacia de la elevación a rango constitucional de los tratados internacionales sobre derechos humanos, en Cuadernos de análisis jurídico (1990) 13.

Protección de datos personales (Santiago, Consejo para la Transparencia, 2011).

Rajevic Mosler, Enrique, Protección de datos y transparencia en la administración pública chilena: Inevitable y deseable ponderación /en/ VV.AA. Reflexiones Sobre el Uso y Abuso de los Datos Personales en Chile (Santiago, Expansiva, 2011).

SAENGER GIANONI, Fernando, Consideraciones para estimar que los tratados en materia de derechos humanos no forman parte de la Constitución, en Revista Chilena de Derecho 20 (1993) 2-3.

SAnZ Salguero, Francisco Javier, Relación entre la protección de los datos personales y el derecho de acceso a la información pública dentro del marco del derecho comparado, en Revista Ius et Praxis 22 (2016) 1.

Sanz Salguero, Francisco Javier, Solicitud de acceso a la información y tutela de los datos personales de un tercero, en Revista de Derecho de la Pontificia Universidad Católica de Valparaíso 41 (2013) 2.

Tórtora Aravena, Hugo, Apuntes derecho constitucional. [visible en Internet: http:// constitucionalchile.blogspot.com/2010/07/02-bases-de-la-institucionalidad.html]. 